\title{
Added Value of Diffusion-Weighted MR Imaging to Non-Enhanced CT in the Evaluation of Acute Appendicitis Oguzhan Ozdemir, ${ }^{1,{ }^{*}}$ Yavuz Metin, ${ }^{1}$ Nurgul Orhan Metin, ${ }^{1}$ Ozlem Bilir, ${ }^{2}$ Ozcan Yavasi, ${ }^{2}$ and Ali Kupeli ${ }^{3}$ \\ ${ }^{1}$ Department of Radiology, Faculty of Medicine, Recep Tayyip Erdogan University, Rize, Turkey \\ ${ }^{2}$ Department of Emergency, Faculty of Medicine, Recep Tayyip Erdoğan University, Rize, Turkey \\ ${ }^{3}$ Department of Radiology, Mus Public Hospital, Mus, Turkey \\ "Corresponding author: Oguzhan Ozdemir, Department of Radiology, Faculty of Medicine, Recep Tayyip Erdogan University, Rize, Turkey. E-mail: droguzrad@hotmail.com
}

Received 2016 August 13; Revised 2017 October 02; Accepted 2017 October 17.

\begin{abstract}
Background: Although non-enhanced CT (NECT) can be applied for the diagnosis of acute appendicitis in obligatory conditions such as impaired renal function, it is not as effective as contrast-enhanced CT.

Objectives: This prospective study aims to determine the added value of diffusion weighted imaging(DWI) in the diagnosis of acute appendicitis when combined with non-enhanced computed tomography (NECT).

Patients and Methods: Between June 2014 and January 2017, 94 patients ( 48 male, 46 female) imaged with NECT and DWI for acute appendicitis were enrolled in this prospective study. DWI was obtained with b factors 0,500 and $1000 \mathrm{~s} / \mathrm{mm}^{2}$, and assessed with visual and quantitative analysis. Results of NECT and DWI, and combined imaging (NECT and DWI) were analyzed by means of sensitivity, specificity and accuracy.

Results: NECT and DWI provided 91.5\% - 94.3\% sensitivity, 82.6\% - 86.9\% specificity and 89.3\% - 92.5\% accuracy, respectively. The combined protocol (NECT and DWI) revealed $98.5 \%$ sensitivity, $95.8 \%$ specificity and $97.8 \%$ accuracy. The addition of DWI to NECT provided a $7 \%, 13.2 \%$ and $8.5 \%$ increase in the sensitivity, specificity and accuracy, respectively. This increase was statistically significant $(\mathrm{P}<$ 0.05).

Conclusion: DWI is an efficient technique for the diagnosis of acute appendicitis. This even may become more efficient if added to a NECT scan. We recommend using DWI when NECT is inevitable for different reasons. It may increase the diagnostic accuracy of NECT to avoid an additional contrast-enhanced CT scan.
\end{abstract}

Keywords: Acute Appendicitis, Computed Tomography, Diffusion-Weighted Imaging, Emergency Department

\section{Background}

Acute abdominal pain is a common presentation in patients who are admitted to an emergency department. Differential diagnosis of acute abdominal pain ranges from mild to life-threatening conditions. The most common causes are acute appendicitis (AA), diverticulitis, cholecystitis and bowel obstruction. Less common causes include perforated viscus and bowel ischemia.

AA is a common surgical emergency, with an estimated lifetime risk of $7 \%$ - $8 \%$. Diagnostic imaging is generally performed following a detailed patient history, physical examination and laboratory tests (1). An accurate and quick diagnosis is essential for appropriate management in emergency department settings. Imaging can change the preliminary diagnosis and decision making about management, or it can increase the level of diagnostic certainty $(2,3)$. Ultrasonography (US) and computed tomography (CT) are the imaging modalities typically used for the pri- mary evaluation of these patients, and CT is used more often than US (4-8). Among these two modalities, CT has been reported to be more sensitive than US, not only for primary diagnosis but also for identifying potential complications $(9,10)$. Despite these advantages, CT use is limited in the pediatric age group and pregnant patients for whom US is more often employed $(11,12)$. In most patients, the CT features of AA include enlargement of the appendix, wall thickening with enhancement, periappendiceal fat stranding, the presence of an appendicolith and in some cases, focal thickening of the terminal ileum or cecum. On $\mathrm{CT}$, traditionally a cut-off value $>6 \mathrm{~mm}$ for the outer diameter of the appendix has been used for the diagnosis of AA. However, several studies have demonstrated that 6 $\mathrm{mm}$ is too low of a cut-off value on cross-sectional imaging, and they have suggested an 8 - 9 mm cut-off value (13). A pericecal phlegmon or abscess strongly suggests perforated appendicitis. However, these findings are nonspecific, and they can also be seen with other disease processes 
involving the cecum and terminal ileum (14). Many clinicians, as in the case of our emergency department practice, avoid contrast-enhanced CT (CECT) due to concerns about contrast media-associated nephrotoxicity or allergic reactions. Although non-enhanced CT (NECT) can be applied for acute abdominal pain in obligatory conditions, such as impaired renal function, it is not as effective as CECT. In some of these cases, repeated examination with contrast administration might be required, which results in an increased radiation dose and a delay in diagnosis.

Several studies have reported the benefits of using magnetic resonance imaging (MRI) techniques that are particularly applicable in an emergency department setting $(15,16)$. Diffusion-weighted magnetic resonance imaging (DWI) of the abdomen and pelvis has been increasingly used since the 1990s with the introduction of stronger diffusion gradients, faster imaging sequences and improvements in MRI hardware. DWI can be performed in a very short time without the use of intravenous (IV) contrast media, allowing for fast, qualitative assessment (17-20) Many previous studies have reported on the use of rapid MRI techniques that are particularly applicable for emergency department settings $(15,16,21-24)$. DWI is an active field of research for this purpose. It provides information related to the cellularity of tissues. Many articles have been published on the contribution of DWI in evaluating the inflammatory and neoplastic diseases of the abdomen $(25,26)$. Recent studies have also proven the effectiveness of DWI in acute abdominal situations $(15,16,21-24,27)$. DWI is reported to have a sensitivity of $99 \%$ and specificity of $97 \%$ in the diagnosis of AA in adults (27).

\section{Objectives}

In the present study, the primary reason for adding DWI was the high number NECT scans performed for acute abdominal pain in emergency situations by emergency department physicians. Because CECT is the gold standard imaging method for AA, when NECT is used, the demand for an additional contrast-enhanced scan (mostly CECT) sometimes seems inevitable. As far as we know, there are no published studies investigating the contribution of DWI to NECT in the diagnosis of AA in emergency department settings. Considering the fact that DWI is a fast and non-invasive technique that is efficient in the diagnosis of acute abdominal pathologies, we added it to the NECT protocol for suitable patients (those with no known contraindications for MRI). Thus, the goal of this prospective study was to assess whether DWI with its ability performing both qualitative and quantitative analysis, by combining to NECT, can improve the diagnostic efficacy to detect an acute infectious-inflammatory process in the appendix, without a demand of CECT, the gold standard imaging method.

\section{Patients and Methods}

\subsection{Study Population}

This prospective study was conducted between June 2014 and January 2017. DWI was used for patients with acute abdominopelvic pain who had to be scanned with NECT. An NECT scan was performed after an initial US examination was inconclusive. During the study period, a total of 362 patients that were scanned with NECT also underwent DWI within a few hours. Among those, we prospectively reviewed the results of a total of 122 consecutive patients (59 males, 63 females; mean age, $36.3 \pm 13.9$ years; age range, 18 - 76 years) based on symptoms and physical examination findings that suggested a clinical diagnosis of AA. Eight of the 122 patients had excessive motion artifacts, five had appendices that were not visible on DWI and 15 had other diagnoses (all with acute right adnexal pathologies confirmed by MRI causing right lower quadrant (RLQ) pain); thus, 28 patients were excluded from the study. Consequently, the study group consisted of 94 patients ( 48 males, 46 females; mean age, $38.1 \pm 13.6$ years; age range, 18 - 76 years). Surgery was the gold standard test for definitive diagnosis. Those who did not undergo surgery by the decision of our surgical team, were decided a threemonth close follow-up. The patients were warned about the possibility of overlooking AA with imaging; they were called for a clinical visit at the end of each month. Pregnant women, children under the age of 16, clinically unstable patients, patients with poor cooperation and those with claustrophobia were excluded from the study. The study was approved by the institutional ethics committee and written consent was obtained from each patient before imaging.

\subsection{Imaging Protocols}

DWI was performed using a 1.5-T MRI unit (Magnetom Aera; Siemens AG, Erlangen, Germany) in supine position with an eight-channel phased-array coil. Axial diffusionweighted single-shot echoplanar imaging (EPI) sequence with fat suppression without breath holding was performed. At the beginning of the examination, a threeplane gradient echo was used as the localizer sequence. The imaging parameters were repetition time (TR)/echo time (TE): 7500/80 ms; section thickness: $5 \mathrm{~mm}$; intersection gap: 30\%; matrix size: $192 \times 192$; number of excitations (NEX): 2; parallel imaging with reduction factor: 2; field of view (FOV): $400 \times 400 \mathrm{~mm}$; acquisition time: approximately 4 min and water excitations with $b$ values of 0 
$\mathrm{s} / \mathrm{mm}^{2}, 500 \mathrm{~s} / \mathrm{mm}^{2}$ and $1000 \mathrm{~s} / \mathrm{mm}^{2}$. With the exception of DWI, no other MRI sequence was obtained for participants in the present study.

Computed tomography was performed with a 16-slice multidetector-row scanner (Toshiba Alexion ${ }^{\mathrm{TM}} /$ Advance; Toshiba Medical Systems Corporation Nashu). The following CT protocol was used: $120 \mathrm{kVp}$, a tube current of 150 $165 \mathrm{mAs}$, maximum $2.5 \mathrm{~mm}$ collimation, a slice thickness of $3 \mathrm{~mm}$ and a $0.5 \mathrm{~s}$ rotation time. Neither an IV contrast agent nor an oral contrast agent was used. The decision about the patients' imaging management at presentation (use of NECT or CECT) was made by the emergency department physicians.

\subsection{Image Analysis}

All the CT and DWI images were evaluated at a dedicated workstation (Syngo.via; Siemens AG, Erlangen, Germany). One radiologist reviewed the NECT-only group. A second radiologist, who was blinded to the NECT results, evaluated the DWI-only group. Both radiologists assessed the NECT and DWI images to reach consensus regarding final diagnosis. The radiologists had at least five years of experience assessing body CT, MRI and emergency radiology. The radiologists were aware of the clinical and laboratory findings as well as the initial imaging methods (e.g. plain radiography and US).

On the NECT images, AA was considered when the appendiceal diameter exceeded $8 \mathrm{~mm}$ and at least one of the following was present: abnormal periappendiceal fat stranding, fluid or abscess.

On the DWI sequences, the appendices were assessed visually and quantitatively as follows:

a. Visual analysis

Visual analysis of the signal intensity was performed on b 1000 images. AA was considered when the appendiceal diameter exceeded $8 \mathrm{~mm}$, and a strong hyperintense signal on DWI and concomitant hypointensity on the apparent diffusion coefficient (ADC) map were observed with or without the following: periappendiceal fat stranding, surrounding fluid or abscess. The signal intensity was evaluated in comparison to the cecum or neighbouring ileum as follows: hyperintense, hypointense or isointense. A strong hyperintense signal was accepted as abnormal. As in the b 1000 images, visual assessment was also performed on the ADC maps in comparison to the cecum or neighboring ileum as follows: hyperintense, hypointense or isointense. A hypointense signal was interpreted as abnormal.

b. Quantitative Analysis

The ADC values were measured using $3 \mathrm{~b}$ factors ( 0 $\mathrm{s} / \mathrm{mm}^{2}, 500 \mathrm{~s} / \mathrm{mm}^{2}$ and $1000 \mathrm{~s} / \mathrm{mm}^{2}$ ). For quantitative analysis of the ADC map, the region of interest(ROI) was placed in the most hypointense part covering the wall and lumen. ROI was inserted three times for each patient, and the mean of these was used as the ADC value. For all patients, the DWI datasets were evaluated at an independent workstation (Syngo.via, Siemens) for post-processing and ADC map analysis.

Finally, both reviewers reassessed the NECT images taking the features on the DWI into account, and consensus on the diagnosis was reached. With the combined NECTDWI imaging protocol, AA was considered when the appendiceal diameter on either modality exceeded $8 \mathrm{~mm}$ with a concomitant strong hyperintense signal on the DWI plus hypointensity on the ADC map with at least one of the following: periappendiceal fat stranding, surrounding fluid or abscess on either modality.

\subsection{Statistical Analysis}

All the data were analyzed using Statistical Package for the social sciences software (SPSS 13.0 Statistical Software, SPSS Inc., Chicago, IL, USA). The median age and age ranges, the NECT versus DWI appendix diameters and the ADC values of the study and control groups were calculated. The Kolmogorov-Smirnov test was used to determine the deviation from normal distribution. The parametric Student's t-test was used to compare the ADC values of the patients in the study and control groups. Furthermore, the Wilcoxon test was used to assess the differences between the diameter of the appendices on NECT and DWI. Sensitivity, specificity, positive predictive value (PPV), negative predictive value (NPV), with their 95\% CI, and the accuracy of NECT and DWI and the combined imaging (NECT-DWI) were calculated. The McNemar test was used to compare the diagnostic performance of NECT and the combined NECT-DWI protocol. A P value of less than 0.05 was considered to indicate a statistically significant difference.

\section{Results}

The demographic features of the study patients are shown in Table 1. NECT scanning was used for the following reason: 19 patients (5.5\%) had renal failure, 35 patients (10.2\%) had previous allergic reactions to contrast agents, 75 patients (21.9\%) did not give consent for the use of contrast agent and the remaining 213 patients (62.2\%) were imaged with NECT based on the decision of emergency department physicians without any contraindication to contrast agent.

The mean and appendiceal diameter ranges for NECT and DWI were $9.1 \pm 1.6$ (range: $5.8-13$ ) and $9.1 \pm 1.6$ (range: $5.9-12.8$ ), respectively. No significant difference in the appendiceal diameter was observed in the NECT and DWI images $(\mathrm{P}=0.67)$. 
Table 1. Patients' Characteristics at Presentation $(n=94)$

\begin{tabular}{lc}
\hline & No. $(\%)$ \\
\hline Gender & \\
\hline Male & $48(51)$ \\
\hline Female & $46(48.9)$ \\
\hline Presentation of patients & \\
\hline Generalized abdominal pain & $8(8.5)$ \\
\hline Epigastric pain & $7(7.4)$ \\
\hline Right lower quadrant pain & $62(65.9)$ \\
\hline Generalized pelvic pain & $17(18)$ \\
\hline Fever & \\
\hline+ & $25(26.5)$ \\
\hline - & $69(73.4)$ \\
\hline High & \\
\hline Low & $76(80.8) / 66(70.2)$ \\
\hline
\end{tabular}

${ }^{\mathrm{z}}$ Abbreviations: CRP, C-reactive protein; WBC, white blood cell count.

Visual analysis of DWI $(\mathrm{n}=94)$ revealed a hyperintense signal on $\mathrm{b} 1000$ images with varying degrees of hypointensity on the ADC map in 70 patients (74.4\%) and an izo-hypointense signal on b 1000 images with varying degrees of hyperintensity on the ADC map in 24 patients (25.5\%). In 71 surgically-proven appendicitis patients, four (5.6\%) had either a hypointense or an isointense signal on $b$ 1000 images with concomitant hyperintensity on the ADC map, reported negative for AA; 67 (94.3\%) patients with AA had a strong hyperintense signal with hypointensity on the ADC map when reviewed with DWI only (Figure1). However, three patients with a hyperintense signal on $b 1000$ images with concomitant hypointensity on the ADC map were found to be normal (one surgically-proven and two according to combined NECT-DWI imaging with clinical and laboratory results and follow-up data), as shown in Figure 2.

A definitive diagnosis could be made in 74 patients (78.7\%) with surgical confirmation and 71 (95.9\%) were proven to have AA. Follow-up of the remaining 20 (21.2\%) non-operated patients was uneventful, without a need of
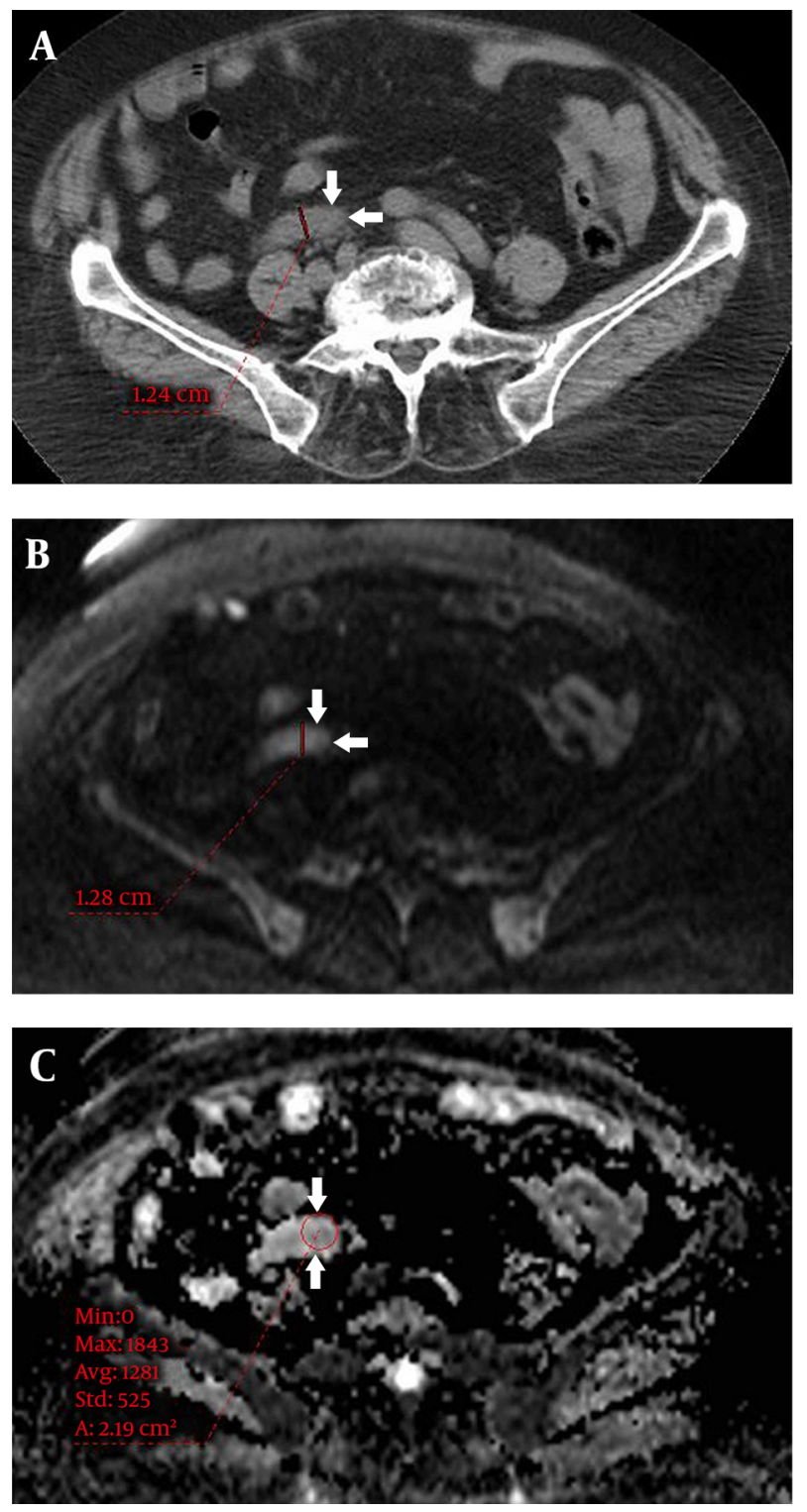

Figure 1. A34-year-old man with acute right lower quadrant pain for a day had acute appendicitis at surgery. A, Non-enhanced computed tomography (NECT) shows enlargement of the appendix with some stranding of surrounding fatty tissue (arrows). B, Diffusion weighted imaging (DWI) clearly depicts an enlarged hyperintense tubular structure (arrows) and C, Apparent diffusion coefficient (ADC) map shows diffusion restriction (arrows).

CECT or any surgical intervention. The ADC values of the 71 surgically-proven appendicitis patients $(n=71)$ and the 23 patients with normal appendices $(n=23$, three surgicallyproven and 20 with normal NECT and DWI results) were: $1.28 \pm 0.31\left(\times 10^{-3} \mathrm{~mm}^{2} / \mathrm{s}\right)$, range: $0.46-1.74\left(\times 10^{-3} \mathrm{~mm}^{2} / \mathrm{s}\right)$; $1.93 \pm 0.19\left(\times 10^{-3} \mathrm{~mm}^{2} / \mathrm{s}\right)$, range: $1.49-2.24\left(\times 10^{-3} \mathrm{~mm}^{2} / \mathrm{s}\right)$, respectively. The ADC values were found to be significantly lower in the surgically-proven patients with AA than those 

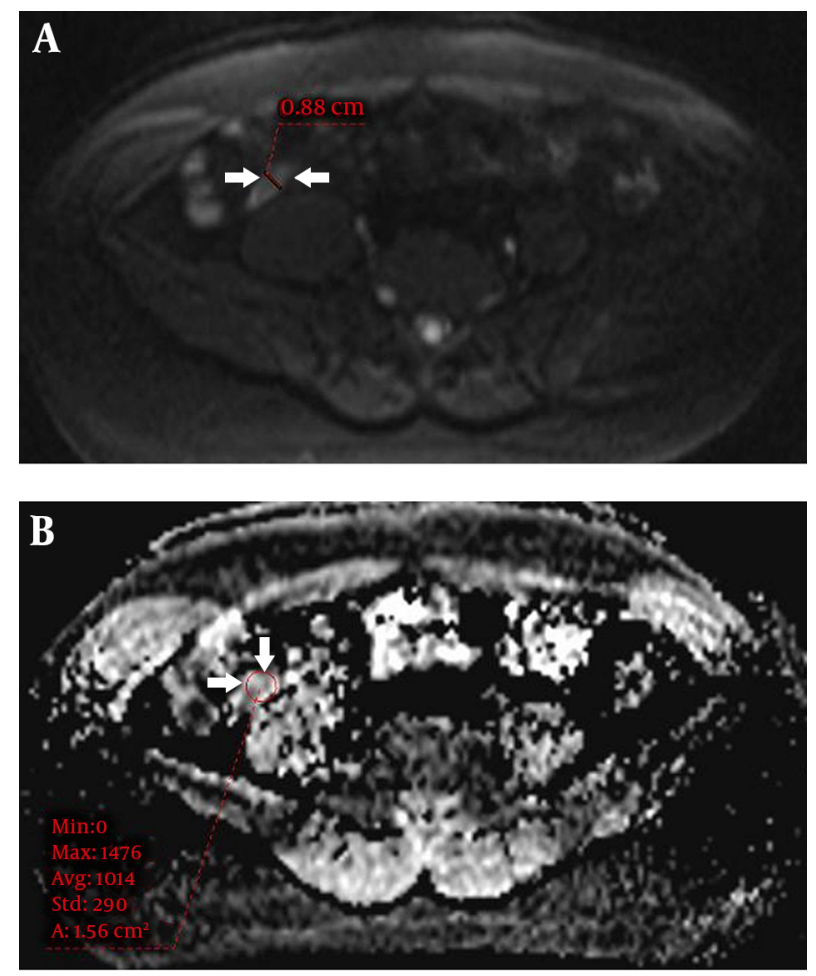

Figure 2. A 18-year-old man with right lower quadrant pain for the last two days. Following surgery, microscopy revealed lymphoid hyperplasia without findings of acute appendicitis. Non-enhanced computed tomography (NECT) (not shown here) shows only slight enlargement of the appendix with fine periappendiceal fatty tissue. Combined imaging report was positive for acute appendicitis. A, Diffusion weighted imaging (DWI) shows enlargement of the appendix with hyperintense signal (arrows) and B, Apparent diffusion coefficient (ADC) map shows restricted diffusion of the appendix (arrows).

with normal appendices $(\mathrm{P}<0.001)$.

The results and diagnostic performance of the imaging modalities are shown in Tables 2 and 3, respectively. The following values were obtained for the NECT-only and the DWI-only protocols: $91.5 \%$ - $94.3 \%$ sensitivity, $82.6 \%$ - $86.9 \%$ specificity and $89.3 \%$ - 92.5\% accuracy, respectively. The values for the combined protocol (NECT-DWI) were: $98.5 \%$ sensitivity, $95.8 \%$ specificity, and $97.8 \%$ accuracy. The addition of DWI provided a $7 \%, 13.2 \%$ and $8.5 \%$ increase in the sensitivity, specificity and accuracy of NECT, respectively (Figure 3). This increase was statistically significant $(\mathrm{P}<0.05)$.

\section{Discussion}

$\mathrm{CT}$ is the most commonly used imaging modality for evaluating acute abdominal pain with a high sensitivity and specificity over $90 \%$. The use of multidetector CT scanners has increased the accuracy rates in the diagnosis of specific disease processes, such as appendicitis and diverticulitis, with acute abdominal pain. In cases with acute
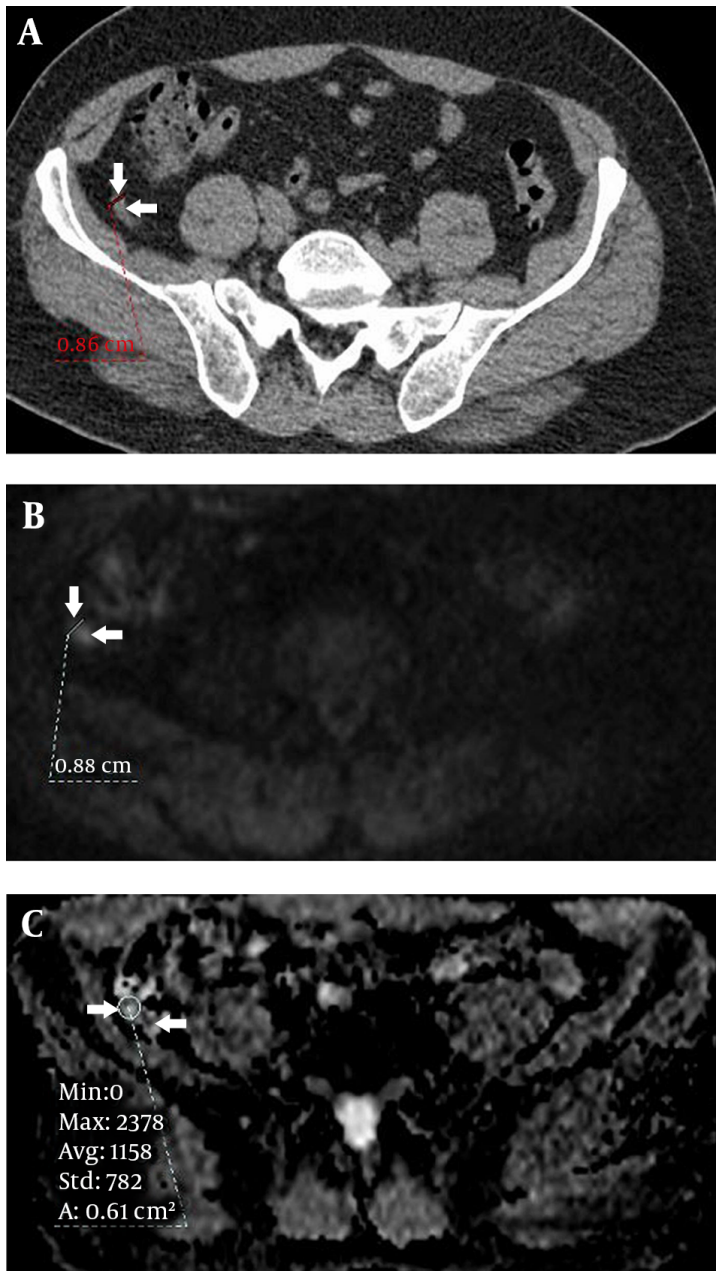

Figure 3. A 37-year-old female patient with right lower quadrant pain for the last two days. Surgery revealed acute appendicitis. A, Non-enhanced computed tomography (NECT) shows a slightly enlarged appendix $(8.6 \mathrm{~mm})$ with fine surrounding fatty tissue planes that was reported as normal by NECT only review (arrows). B, Diffusion weighted imaging (DWI) depicts a strong hyperintense signal of the enlarged appendix (arrows) with some periappendiceal fat blurring, and C, Apparent diffusion coefficient (ADC) map reveals diffusion restriction (arrows).

abdominal pain, scanning of the entire abdomen using IV administration of an iodinated contrast agent is recommended. Although abdominal CT can be performed without a contrast agent, it is reported that CECT has better accuracy rates; for example, a positive predictive value of $95 \%$ has been reported for the diagnosis of AA (18). Today, with the development of fast imaging techniques, MRI has become an important imaging method for evaluating acute abdominal pain. DWI is an active field of research for this purpose.

In some clinical practices, NECT is frequently used to image acute abdominal pain despite the absence of any contraindication to contrast use. In our clinical practice, 
Table 2. Results of Imaging Modalities

\begin{tabular}{lcc}
\hline Patients & No & Mean \pm SD \\
\hline ADia in NECT review & 94 & $9.1 \pm 1.6$ (range, $5.8-13 \mathrm{~mm})$ \\
\hline ADia in DWI review & 94 & $9.1 \pm 1.6$ (range, $5.9-12.8 \mathrm{~mm})$ \\
\hline ADC value in AA patients & 71 & $1.28 \pm 0.31$ \\
\hline ADC value in normal patients & 23 & $1.93 \pm 0.19$ \\
\hline Signal intensity on DWI $(\mathbf{b}=\mathbf{1 0 0 0})$ & & $74.4 \%$ \\
\hline \multicolumn{1}{c}{ Hyperintense } & $70\left(67^{\mathrm{a}}\right)$ & $25.5 \%$ \\
\hline \multicolumn{1}{c}{ Hypo-or isointense } & $24\left(4^{\mathrm{b}}\right)$ & \\
\hline
\end{tabular}

Abbreviations: AA, acute appendicitis; ADC, apparent diffusion coefficient; ADia, appendiceal diameter; DWI, diffusion weighted imaging; NECT, non-enhanced computed tomography.

${ }^{a}$ True positive.

${ }^{\mathrm{b}}$ False negative.

Table 3. Diagnostic Efficacy of NECT, DWI and Combined Imaging (NECT and DWI)

\begin{tabular}{|c|c|c|c|}
\hline & NECT & DWI & NECT and DWI \\
\hline True-positive (No. of lesions) & 65 & 67 & 70 \\
\hline True-negative (No. of lesions) & 19 & 20 & 22 \\
\hline False-positive (No. of lesions) & 4 & 3 & 1 \\
\hline False-negative (No. of lesions) & 6 & 4 & 1 \\
\hline Sensitivity ${ }^{a}$ & $91.5(82.5-96.8)$ & $94.3(86.2-98.4)$ & $98.5(92.4-100.0)$ \\
\hline Specificity $^{a}$ & $82.6(61.2-95.0)$ & $86.9(66.4-97.2)$ & $95.8(78.1$ - 99.9) \\
\hline $\mathbf{P P V}^{\mathbf{a}}$ & $94.2(86.9-97.5)$ & $95.7(88.6-98.5)$ & $98.5(91.1-99.8)$ \\
\hline $\mathbf{N P V}^{\mathbf{a}}$ & $76.0(59.0-87.4)$ & $83.3(65.6-92.9)$ & $95.8(75.8-99.4)$ \\
\hline Accuracy, \% & 89.3 & 92.5 & 97.8 \\
\hline
\end{tabular}

Abbreviations: CI, confidence interval; DWI, diffusion weighted imaging; NECT, non-enhanced computed tomography; NPV, negative predictive value; PPV, positive predictive value.

${ }^{\mathrm{a}}$ Values are expressed as (\%) (\%95 CI).

this is mainly due to the preferences of emergency department physicians, particularly when acute abdominal pain is observed after regular working hours when no, or a limited number of, radiologists and/or other radiology staff are on active duty.

Initial US as a diagnostic strategy in acute abdominal pain before examination with CT can reduce unnecessary
CT scans and radiation exposure $(8,28,29)$. In our study, all the patients underwent US examination before NECT or DWI. A CT scan was performed when the US findings were inconclusive.

Visual assessment of DWI revealed a hyperintense signal in 67 (94.3\%) of 71 surgically-proven appendicitis patients. This result demonstrates that DWI may help dif- 
ferentiate the hyperintense tubular signal from the hypointense background. In addition to visual evaluation of DWI, simultaneous quantitative analysis using an ADC map may show diffusion restriction in the inflammatoryinfectious process, as in the case of $\mathrm{AA}(30,31)$. The results of the present study showed that the mean ADC values were significantly higher in the surgically-proven appendicitis patients than the normal patients $(\mathrm{P}<0.001)$. With the advantage of previously-performed NECT, combined NECTDWI imaging may significantly increase the diagnostic efficacy, as shown in our study.

In this present study, a review of NECT-only, DWI-only and combined NECT-DWI imaging for the diagnosis of AA had sensitivity, specificity and accuracy rates of $91.5 \%, 82.6 \%$ and $89.3 \%, 94.3 \%, 86.9 \%$ and $92.5 \%$ and $98.5 \%, 95.8 \%$ and $97.8 \%$, respectively. Hence, the combination of DWI and NECT significantly increased the diagnostic efficacies of these protocols $(\mathrm{P}<0.05)$.

The sensitivity and specificity of NECT for appendicitis is reported to be $90 \%$ - 95\%, while it is closer to $99 \%$ with CECT (29). Our results are compatible with the findings reported in the literature. In the present study, the addition of DWI increased the diagnostic efficacy of NECT to the efficacy of CECT reported in the literature (29). In all the patients $(n=94)$, there was no need for a CECT scan for final diagnosis, based on the decision of both radiologists that reviewed the scans and the emergency department physicians. Only 74 patients (78.7\%) were surgically confirmed (definitive diagnosis), while the diagnoses of 20 patients $(21.2 \%)$ were not confirmed by a gold standard imaging method that is CECT in this case. However, in addition to the normal imaging findings, and without a CECT scan, physical examination and laboratory based findings and three-month follow-up data did not favor AA in these patients. It seems that DWI not only has the potential to improve the diagnostic performance of NECT so that it is similar to CECT, it may also help avoid the use of repeated CECT scans for final diagnosis.

At low b-values, DWI is more similar to a T2-weighted image. Hence, the absence of T2 images was not a problem in our study. We did encounter problems caused by low spatial resolution of the diffusion images, low signalto-noise ratio (SNR) and artifacts related to motion (e.g. respiration, arterial beating and bowel movements). Since DWI does not require any contrast agent and the images can be obtained in a short amount of time with ultrafast sequences, we tried to minimize these problems with repeated scans. These ultrafast EPI sequences can collect data within $30-60$ msec. Thus, most of the problems related to movement artifacts were eliminated. Problems with low spatial resolution of DWI restricted the appearance of anatomical details in most of the images, especially at high b-values (e.g. $1000 \mathrm{~s} / \mathrm{mm}^{2}$ ) in which SNR decreases. Therefore, we used low b-value (e.g. $0 \mathrm{~s} / \mathrm{mm}^{2}$ or $500 \mathrm{~s} / \mathrm{mm}^{2}$ ) images in which the DWI images resembled T2 images. This helped in the visualization of anatomical details. Furthermore, it is important to note that we used NECT images as basic images, which also helped us obtain anatomical details.

The present study has a number of limitations. First, it is a non-randomized study in which unstable patients and patients with poor cooperation were excluded. Moreover, we only reviewed the results of patients with a clinical suspicion of AA among patients with acute abdominopelvic pain who underwent NECT followed shortly by DWI. Second, since the final diagnosis was made only after two radiologists reached consensus (reviewing the NECT and DWI images), inter-observer variability was not evaluated. Third, even though DWI is helpful in evaluating acute abdominal pathologies, and when added to NECT without contrast administration it provides as much diagnostic accuracy as CECT, patients have to be imaged using two different imaging techniques. In the non-surgical patient group ( $n=20)$, no definitive diagnostic test was used, unlike the surgical group in which a surgical confirmation of the final diagnosis was achieved. Instead, management of the patients in the non-surgical group relied on the combined NECT-DWI scanning, clinical and laboratory results and three months of close follow-up data. Furthermore, we did not perform quantitative analysis of the signal intensities at different $b$ values, which might increase the diagnostic efficacy. Fourth, an extra DWI would not be cost-effective and MRI is not available at all institutions; it might be impractical to perform DWI in all causes of acute abdominal pain. Last, we did not include other MRI sequences that provide morphological information, which could have better identified the lesion borders, especially with the use of IV contrast. However, the inclusion of other MRI sequences would not address the aim of this study because we tried to implement the fastest MRI method without the use of IV contrast media in emergency department conditions. Further studies on the use of different imaging protocols, such as fast MRI sequences combined with DWI, are also required.

In conclusion, CECT is a well-known gold standard imaging modality used to evaluate AA. In some patients or physician-related conditions, non-enhanced imaging might be preferred. At that point, combining DWI with NECT might aid in the detection of the inflammation or infection, and it could increase the diagnostic accuracy to a level that is comparable to CECT. Consequently, it might be possible to reduce the number of additional CECT scans that could be necessary for an eventual diagnosis. Therefore, in clinical suspicion of AA, if NECT is used, we pro- 
pose the addition of DWI. Furthermore, we also propose that DWI, as well as new, faster MRI sequences without the need of contrast agents, could be used to diagnosis acute abdominal pain, such as occurs in acute cerebrovascular disease, in emergency department settings. This study's design is preliminary; further studies with larger patient groups with different b-values are needed to clearly document the effectiveness of DWI.

\section{Footnotes}

Conflicts of Interest: There are no known conflicts of interest associated with this publication.

Funding/Support: There has been no significant for this work that could have influenced its outcome.

\section{References}

1. Cartwright SL, Knudson MP. Diagnostic imaging of acute abdominal pain in adults. Am Fam Physician. 2015;91(7):452-9. [PubMed: 25884745].

2. Singh A, Danrad R, Hahn PF, Blake MA, Mueller PR, Novelline RA. MR imaging of the acute abdomen and pelvis: acute appendicitis and beyond. Radiographics. 2007;27(5):1419-31. doi: 10.1148/rg.275065021. [PubMed: 17848700].

3. Pedrosa I, Rofsky NM. MR imaging in abdominal emergencies. Radiol Clin North Am. 2003;41(6):1243-73. [PubMed: 14661669].

4. Lane JS, Schmit PJ, Chandler CF, Bennion RS, Thompson JJ. Ileocecectomy is definitive treatment for advanced appendicitis. Am Surg. 2001;67(12):1117-22. [PubMed: 11768813].

5. Thompson JJ, Bennion RS, Schmit PJ, Hiyama DT. Cecectomy for complicated appendicitis. J Am Coll Surg. 1994;179(2):135-8. [PubMed: 8044380].

6. Bixby SD, Lucey BC, Soto JA, Theysohn JM, Ozonoff A, Varghese JC. Perforated versus nonperforated acute appendicitis: accuracy of multidetector CT detection. Radiology. 2006;241(3):780-6. doi: 10.1148/radiol.2413051896. [PubMed: 17114626].

7. Drake FT, Florence MG, Johnson MG, Jurkovich GJ, Kwon S, Schmidt Z, et al. Progress in the diagnosis of appendicitis: a report from Washington State's Surgical Care and Outcomes Assessment Program. Ann Surg. 2012;256(4):586-94. doi: 10.1097/SLA.0b013e31826a9602. [PubMed: 22964731].

8. Humes DJ, Simpson J. Acute appendicitis. BMJ. 2006;333(7567):530-4. doi: 10.1136/bmj.38940.664363.AE. [PubMed: 16960208].

9. Puylaert JB. Ultrasonography of the acute abdomen: gastrointestinal conditions. Radiol Clin North Am. 2003;41(6):1227-42. vii. [PubMed: 14661668].

10. Doria AS, Moineddin R, Kellenberger CJ, Epelman M, Beyene J, Schuh S, et al. US or CT for Diagnosis of Appendicitis in Children and Adults? A Meta-Analysis. Radiology. 2006;241(1):83-94. doi: 10.1148/radiol.2411050913. [PubMed: 16928974].

11. Levine CD, Aizenstein O, Wachsberg RH. Pitfalls in the CT diagnosis of appendicitis. BrJRadiol.2004;77(921):792-9. doi:10.1259/bjr/95663370. [PubMed: 15447972].

12. Kim K, Kim YH, Kim SY, Kim S, Lee YJ, Kim KP, et al. Low-dose abdominal CT for evaluating suspected appendicitis. $N$ Engl $J$ Med. 2012;366(17):1596-605. doi: 10.1056/NEJMoa1110734. [PubMed: 22533576].
13. Ives EP, Sung S, McCue P, Durrani H, Halpern EJ. Independent predictors of acute appendicitis on CT with pathologic correlation. Acad Radiol. 2008;15(8):996-1003. doi: 10.1016/j.acra.2008.02.009. [PubMed: 18620120].

14. Kim HC, Yang DM, Jin W, Park SJ. Added diagnostic value of multiplanar reformation of multidetector CT data in patients with suspected appendicitis. Radiographics. 2008;28(2):393-405. discussion 405-6. doi: 10.1148/rg.282075039. [PubMed: 18349447].

15. Moore WA, Khatri G, Madhuranthakam AJ, Sims RD, Pedrosa I. Added value of diffusion-weighted acquisitions in MRI of the abdomen and pelvis. AJR Am J Roentgenol. 2014;202(5):995-1006. doi: 10.2214/AJR.12.9563. [PubMed: 24758652].

16. Koh DM, Collins DJ. Diffusion-weighted MRI in the body: applications and challenges in oncology. AJR Am J Roentgenol. 2007;188(6):1622-35. doi: 10.2214/AJR.06.1403. [PubMed: 17515386].

17. Rathod SB, Kumbhar SS, Nanivadekar A, Aman K. Role of diffusionweighted MRI in acute pyelonephritis: a prospective study. Acto Radiol. 2015;56(2):244-9. doi: 10.1177/0284185114520862. [PubMed: 24443116].

18. Stoker J, van Randen A, Lameris W, Boermeester MA. Imaging patients with acute abdominal pain. Radiology. 2009;253(1):31-46. doi 10.1148/radiol.2531090302. [PubMed: 19789254].

19. Bruegel M, Gaa J, Waldt S, Woertler K, Holzapfel K, Kiefer B, et al. Diagnosis of hepatic metastasis: comparison of respirationtriggered diffusion-weighted echo-planar MRI and five t2-weighted turbo spin-echo sequences. AJR Am J Roentgenol. 2008;191(5):1421-9. doi: 10.2214/AJR.07.3279. [PubMed: 18941080].

20. Sumi M, Van Cauteren M, Nakamura T. MR microimaging of benign and malignant nodes in the neck. AJR Am J Roentgenol. 2006;186(3):749-57. doi: 10.2214/A]R.04.1832. [PubMed: 16498102].

21. Lubarsky M, Kalb B, Sharma P, Keim SM, Martin DR. MR imag ing for acute nontraumatic abdominopelvic pain: rationale and practical considerations. Radiographics. 2013;33(2):313-37. doi 10.1148/rg.332125116. [PubMed: 23479698].

22. Qayyum A. Diffusion-weighted imaging in the abdomen and pelvis: concepts and applications. Radiographics. 2009;29(6):1797-810. doi 10.1148/rg.296095521. [PubMed: 19959522].

23. Le Bihan D, Breton E, Lallemand D, Aubin ML, Vignaud J, Laval-Jeantet M. Separation of diffusion and perfusion in intravoxel incoherent motion MR imaging. Radiology. 1988;168(2):497-505. doi: 10.1148/radiology.168.2.3393671. [PubMed: 3393671].

24. Oto A, Zhu F, Kulkarni K, Karczmar GS, Turner JR, Rubin D. Evaluation of diffusion-weighted MR imaging for detection of bowel inflammation in patients with Crohn's disease. Acad Radiol.2009;16(5):597-603. doi: 10.1016/j.acra.2008.11.009. [PubMed: 19282206].

25. Kwee TC, Takahara T, Ochiai R, Nievelstein RA, Luijten PR. Diffusionweighted whole-body imaging with background body signal suppression (DWIBS): features and potential applications in oncology. Eur Radiol. 2008;18(9):1937-52. doi: 10.1007/s00330-008-0968-z. [PubMed: 18446344].

26. Muraoka N, Uematsu H, Kimura H, Imamura Y, Fujiwara Y, Murakami $M$, et al. Apparent diffusion coefficient in pancreatic cancer: characterization and histopathological correlations. J Magn Reson Imaging. 2008;27(6):1302-8. doi: 10.1002/jmri.21340. [PubMed: 18504750].

27. Inci E, Kilickesmez O, Hocaoglu E, Aydin S, Bayramoglu S, Cimilli T Utility of diffusion-weighted imaging in the diagnosis of acute appendicitis. Eur Radiol. 2011;21(4):768-75. doi: 10.1007/s00330-010-19816. [PubMed: 20924585].

28. Atema JJ, van Rossem CC, Leeuwenburgh MM, Stoker J, Boermeester MA. Scoring system to distinguish uncomplicated from complicated acute appendicitis. BrJSurg. 2015;102(8):979-90. doi:10.1002/bjs.9835. [PubMed: 25963411].

29. Pickhardt PJ, Lawrence EM, Pooler BD, Bruce RJ. Diagnostic performance of multidetector computed tomography for suspected acute appendicitis. Ann Intern Med. 2011;154(12):789-96. W-291. doi: 10.7326/0003-4819-154-12-201106210-00006. [PubMed: 21690593]. 
30. Kato H, Kanematsu M, Uchiyama M, Yano R, Furui T, Morishige K. Diffusion-weighted imaging of ovarian torsion: usefulness of apparent diffusion coefficient (ADC) values for the detection of hemorrhagic infarction. Magn Reson Med Sci. 2014;13(1):39-44. [PubMed: 24492742].
31. Lee NK, Kim S, Kim KH, Suh DS, Kim TU, Han GJ, et al. Diffusionweighted magnetic resonance imaging in the differentiation of endometriomas from hemorrhagic cysts in the ovary. Acta Radiol. 2016;57(8):998-1005. doi: 10.1177/0284185115609805. [PubMed: 26543051]. 